\title{
The Role of Multi-Hit Detection Events on the Accurate Measurement of Boron in Atom Probe Tomography
}

\author{
Frederick Meisenkothen ${ }^{1}$, Ty J. Prosa ${ }^{2}$, Eric B. Steel ${ }^{1}$, and R. Prakash Kolli ${ }^{3}$ \\ ${ }^{1}$ Materials Measurement Science Division, National Institute of Standards and Technology, \\ Gaithersburg, U.S.A. \\ ${ }^{2}$ CAMECA Instruments, Inc., Madison, U.S.A. \\ ${ }^{3}$ Department of Materials Science and Engineering, University of Maryland, College Park, U.S.A.
}

In atom probe tomography (APT), some elements tend to field evaporate preferentially in multi-hit detection events [1], where more than one ion is detected between pulses. Boron is one such element, and it is thought that a large fraction of the boron signal may be lost during data acquisition and not reported in the mass spectrum or the 3-D APT reconstruction. Understanding the relationship between the field evaporation behavior of boron and the limitations for detecting multi-hit events should provide insight into the signal loss mechanism for boron and suggest ways to improve boron detection accuracy.

APT specimens were fabricated from nominally pure boron and boron implanted silicon (NIST-SRM2137). A dual-beam FIB/SEM instrument with an in-situ lift-out system was used to prepare APT specimen tips on prefabricated silicon micro-tip arrays [2]. The APT data acquisition was performed in a LEAP 4000X-Si instrument [3] operating in laser-pulsed mode with laser pulse energies of 20-30 pJ and a specimen base temperature of $40 \mathrm{~K}$. The Cameca IVAS software was used to partition the ion data into single-hit and multi-hit detection events. Custom MATLAB scripts were used to extract the event multiplicity (i.e. the number of ions within a given multi-hit event) and the constituent ion information for each multi-hit event.

The mass spectra for the single-hit and multi-hit event data are markedly different, as indicated by the charge-state ratios and the isotope ratios from a nominally pure boron sample shown in Figures 1(a)-(b). For the nominally pure boron specimens, less than $40 \%$ of all detection events were multi-hit events, Figure 2(a), however approximately $60 \%$ of all recorded ions, and about $70 \%$ of the ranged boron ions, were detected within these multi-hit events. For the boron implanted silicon material, less than $3 \%$ of the total detection events were multi-hit events, yet similar to the nominally pure boron specimens, almost $70 \%$ of the detected boron signal was contained within the multi-hit events. Ion correlation analysis [4], Figure 2(b), was used to graphically demonstrate and estimate the detector dead space-time effect (i.e. when ions arrive too closely together in space or in time to be differentiated). This effect is evidenced by the presence of a depleted data zone that is about $0.34 \mathrm{Da}$ wide at $5 \mathrm{Da}$, or $\sim 6 \mathrm{~ns}$ at $6500 \mathrm{~V}$, and is located above the red, $y=x$, line on the histogram. The dead space-time effect on the histogram shows that few same-same multi-hit events (e.g. ${ }^{10} \mathrm{~B}^{+} /{ }^{10} \mathrm{~B}^{+}$), which would fall on the $\mathrm{y}=\mathrm{x}$ line, are recorded. Therefore, multiplicity-2 events consisting of ${ }^{10} \mathrm{~B} /{ }^{10} \mathrm{~B}$ and ${ }^{11} \mathrm{~B} /{ }^{11} \mathrm{~B}$ pairs will be significantly under-represented in the data. A first-order estimate, based on known natural isotopic abundances of boron and the probability of ${ }^{10} \mathrm{~B} /{ }^{10} \mathrm{~B}$ and ${ }^{11} \mathrm{~B} /{ }^{11} \mathrm{~B}$ pairs occurring in multiplicity-2 events, shows that the dead space-time effect could represent a loss or mis-classification of up to $68 \%$ of the multiplicity-2 events in nominally pure boron. Based on the known isotopic abundance and the number of ${ }^{10} \mathrm{~B} /{ }^{11} \mathrm{~B}$ pairs that were detected in boron multiplicity-2 events, this can represent a possible loss of approximately $30 \%$ of the total boron signal [5]. 


\section{References:}

[1] B. Gault, et al., Atom Probe Microscopy, (Springer, NY), 2012, p. 223.

[2] D.J. Larson et al., Handbook of Instrumentation and Techniques for Semiconductor Nanostructure Characterization, London: World Scientific Publishing, Vol. 2 (2011) 407.

[3] Certain commercial equipment, instruments, or materials are identified in this paper in order to specify the experimental procedure adequately. Such identification is not intended to imply recommendation or endorsement by the National Institute of Standards and Technology, nor is it intended to imply that the materials or equipment identified are necessarily the best available for the purpose.

[4] D.W. Saxey, Ultramicroscopy, 111, 2011, p. 473.

[5] The authors would like to thank Dr. Karen Henry of Intel Corporation for her continued interest in this project. We also extend our appreciation to Dr. Doug Meier of NIST for providing some of the specimen materials used in this study.
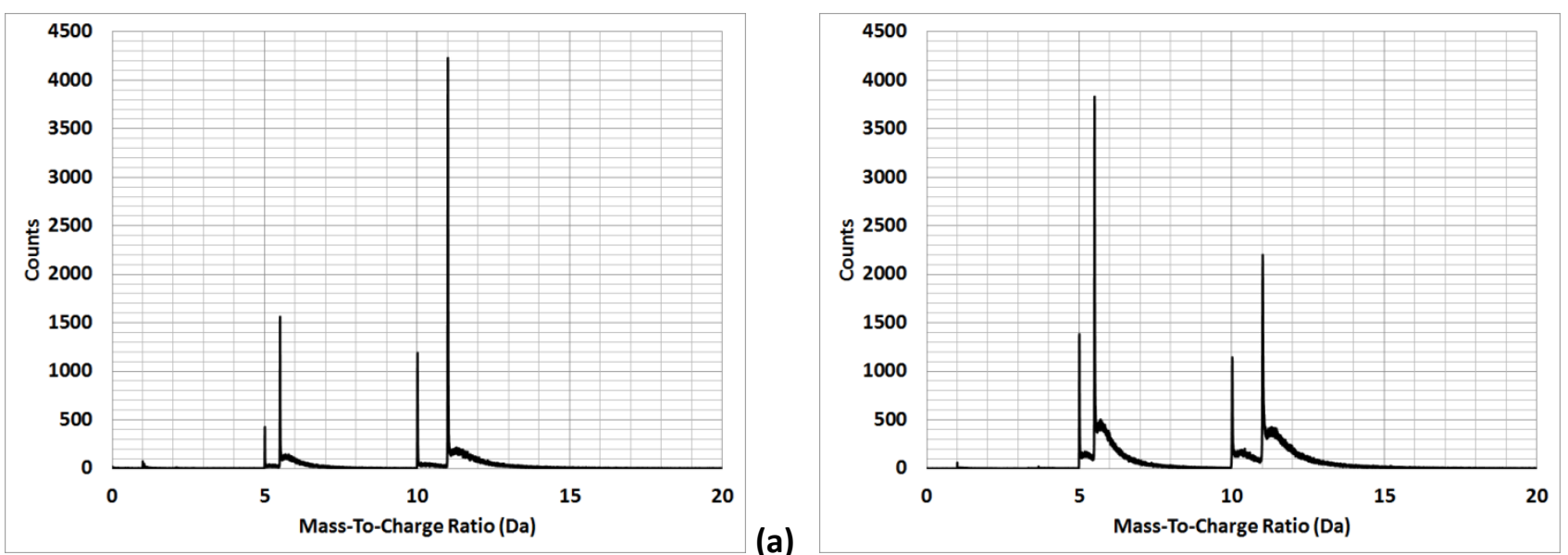

(b)

Figure 1. Nominally pure boron mass spectra for (a) single-hit events, and (b) multi-hit events.

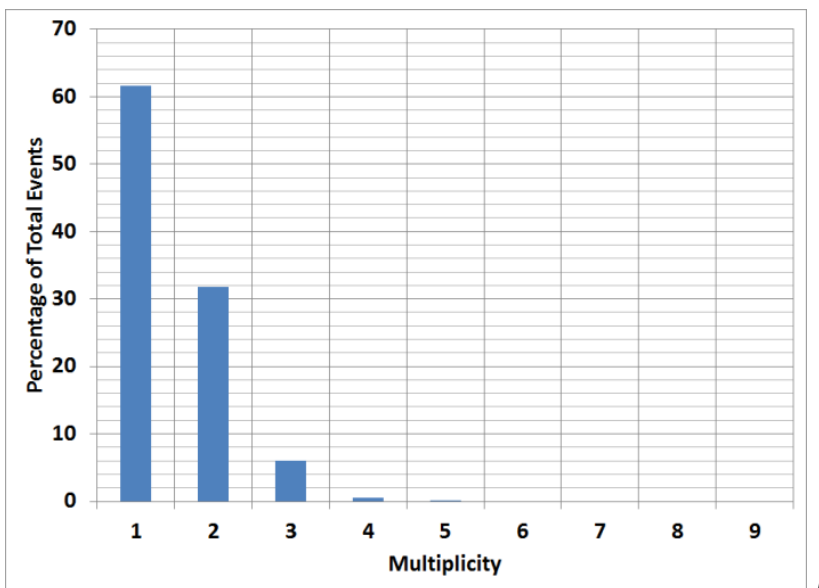

(a)

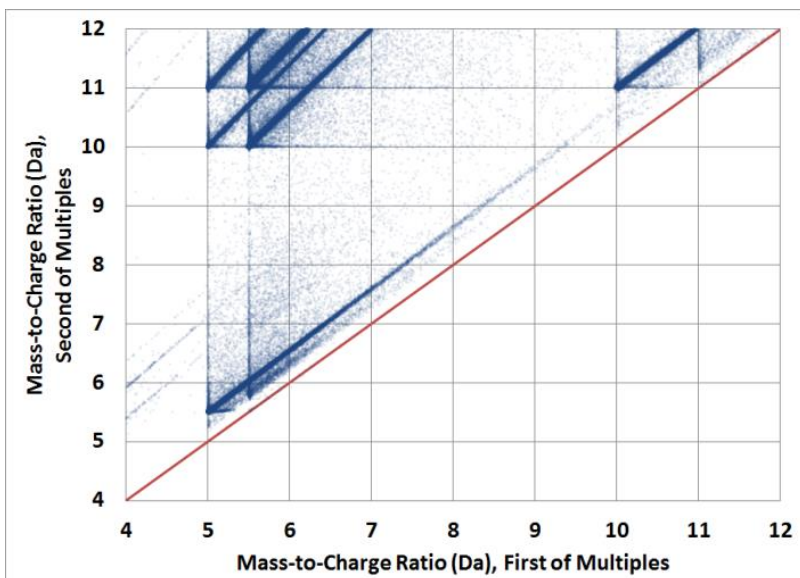

(b)

Figure 2. Nominally pure boron (a) detection event multiplicity, and (b) ion correlation histogram. 